\title{
Smart material actuators as a contributor for IoT-based smart applications and systems: Analyzing prototype and process measurement data of shape memory actuators for reliability and risk prognosis
}

\author{
Philipp HEß $\beta^{*}$ and Stefan BRACKE* \\ * University of Wuppertal, Chair of Reliability Engineering and Risk Analytics \\ Gaußstraße 20, 42119 Wuppertal, Germany \\ E-mail: bracke@uni-wuppertal.de
}

Received: 2 July 2019; Revised: 5 September 2019; Accepted: 24 October 2019

\begin{abstract}
In times of smart products and systems, the Internet of Things (IoT) plays an increasingly important role. IoT combines the digital world (internet) with the physical world (sensors, actuators, robots, smartphones, connected cars etc.). The autonomous operation and remote control of smart systems (e.g. smart home, production hall or line) requires efficient and specially reliable actuators and control mechanisms. Shape memory actuators are particularly suitable for this application due to their properties as they are lightweight, small, energy-efficient and can also be used as sensors at the same time. Many shape memory actuators have been developed for various applications over the past years. Despite great interest, there are no standardized test programs available. The complexity of the shape memory technology is a major challenge in testing fatigue and degradation behavior of components to determine reliability. This article presents fatigue test results of a laboratory test rig of a case study for a shape memory actuator, including all boundary conditions and test requirements. The measurement data consists of different parameters e.g. the stroke of the actuator, the electrical voltage and current (to activate the actuator) as well as the ambient temperature. Since the study comprises only a few prototypes, parametric methods are not suitable for a comprehensive evaluation, therefore parameter-free methods are used as well. The analysis regarding the description of dependencies between the recorded signals and the detection of degradation of the shape memory actuators is discussed in detail. The main objective is the development of a prognosis algorithm in order to be able to predict the failure behaviour of the actuators at an early stage. The methodical approach includes various methods and procedures, which are applied in a logical order. The statistical analytics used in this study are focusing on nonparametric significance tests, such as the Levene's test and the u-test by Wilcoxon and Mann-Whitney. Further methods are the correlation analysis and the regression analysis as well as multivariate 3D-plots. The fundamentals of shape memory alloys, as well as the used statistical nonparametric methods, are described briefly. Finally, the realization (application of the analysis methods) based on the real test rig data of a case study consisting of 18 actuators is shown and discussed in detail.
\end{abstract}

Keywords : Smart material alloy, Smart material actuator, Shape memory alloy, Shape memory actuator, Nonparametric statistics, Degradation analysis, Regression analysis, Reliability analysis, Lifetime estimation

\section{Introduction}

Mechatronic and electromechanical components, systems and assemblies are becoming increasingly important for all products in the investment and consumer goods sectors. The increasing distribution covers all areas of technical complex components and products, where quality is perceived by the customer, in particular through its reliability and functionality. Due to the expanded potential for designing new functionalities, the significance e.g. for automotive engineering has risen sharply. The introduction of mechatronic and electromechanical components began with the implementation of 
electronic anti-lock braking systems in 1979 (Bertsche (2009)). In addition, requirements regarding comfort and safety are playing an important role, which also results in the increased use of actuators and switches in order to realize comfort and safety functions. Furthermore, new technologies and principles (e.g. new materials or material combinations) are being developed to meet the increasing requirements. Table 1 displays typical actuating frequencies of automotive components. It clearly shows that there is a wide range of required cycles for different components.

Table 1 Actuation frequency of automotive components and aggregates

\begin{tabular}{r|c|c}
\hline Component or aggregate & Normal operation & city operation \\
\hline hood & 2.000 & 4.000 \\
\hline Turn signal & 600.000 & 2.000 .000 \\
\hline Gearbox (e.g. 1st gear) & 75.000 & 150.000 \\
\hline Clutch & 330.000 & 630.000 \\
\hline Foot brake & 200.000 & 450.000 \\
\hline Driver's door & 38.000 & 77.000 \\
\hline Handbrake & 8.000 & 18.000 \\
\hline Tailgate & 5.000 & 10.000 \\
\hline Starter & 16.000 & 30.000 \\
\hline Paurce: VDA (2000) & 17.000 & 33.000 \\
\hline
\end{tabular}

Source: VDA (2000)

In contrast to purely mechanical components, electromechanical or mechatronic components are potentially subject to more complex damage scenarios, which is refered to the combination and interaction of mechanical and electrical aspects. As a result, the determination of the degradation behavior is becoming more and more complex. This article focuses on shape memory actuators (smart material actuators, smart actuators) that have an even more complex fatigue behavior than conventional (electrical) actuators, but must have the same lifetime. The main problem is the much longer time needed to test these actuators. Actuation frequencies of more than 100.000 cycles, for example, which are frequently required not only in the automotive sector, must be tested during the development phase. The fatigue test of 100.000 cycles of a conventional actuator that just needs several days, may take months of time regarding a smart material actuator. The testing of actuators and components is carried out by means of fatigue tests (endurance tests) in which the actuator is cyclically loaded until failure occurence. The tests can be performed normally (unaccelerated) or accelerated under stress. Cyclic loading (without pauses) can be a possible accelerated test procedure, which can be used to reduce the required testing time (Nelson (2004) and Meeker (1998)). In case of shape memory actuators a replicable determination of the fatigue behavior, degradation, reliability and service life is not possible due to the lack of standardized test programs including test rigs and test plans (Heß and Bracke (2019)). Despite those major issues, many smart actuators have been developed and tested for varoius applications. According to Elahinia (2016), Heß and Bracke (2018), Heß and Bracke (2019), Hinz et al. (2018), Janocha (2010) and Lagoudas (2008) smart material alloys are used in various aerospace, automotive, biomedical, civil and electrical engineering as well as mechanical applications.

In times of smart products and systems, the Internet of Things (IoT) plays an increasingly important role. The IoT combines the physical world (e.g. actuators, sensors, robots, smartphones, connected cars) with the digital world (internet) (Kouicem et al. 2017). Among other things, this enables the simulation of entire factories (Industry 4.0), control and adjustment of complex systems for example via motors and controllers as well as process monitoring during operation (condition monitoring). In addition to mechanical components, IoT products are characterized by control units, data recording and transmission. The development of new IoT-based systems - which will replace the classic stand-alone solutions - is characterized by the reduction of mechanical components and the increased use of condition monitoring components (control units, sensors, actuators, software etc.). For this purpose, actuators with a high efficiency and reliability are required for various reasons: On the one hand the autonomous operation and remote control of smart systems (e.g. smart home, production hall or line) (Kouicem et al. 2017) and on the other hand the user who perceives the quality through the reliability of the system now becomes the focus of the system itself (Sendler 2019). The connection of several components to (sub)systems and the recording of all environmental influences as well as environmental conditions generates large amounts of data (big data) which can be stored directly in a cloud. In this case, the autonomous and complex interaction of the individual components becomes possible, whereby it is elementary that each individual component - in this example a (smart material) actuator - is reliable. Shape memory actuators are particularly suitable for the application in IoT-based smart systems due to their properties as they are lightweight, small, energy-efficient and can be used as sensors at the same time. But, as mentioned above, there are no standardized test programs, i.e. all actuators available on 
the market have been tested with different test benches. Therefore, this article describes the evaluation of test bench data by means of a case study. For this purpose, the fundamentals of smart material alloys (SMAs) and statistical methods, the test rig and actuators of the study are described in detail. The same parameters, which are monitored and analyzed during prototype testing of shape memory actuators (cf. the case study) in order to determine the long-term reliability, are monitored in an IoT environment during subsequent field operation.

\section{Goal of research work}

The goal of the research work is the proof of the long term reliability of shape memory actuators based on a small amount of prototypes. The reliability of these smart actuators is characterized by the long-term operational capability based on a defined target value regarding the variable "operation cycle". The centerpiece of the actuators is a shape memory alloy element for example a spring, an alloy sheet or - in the case study - a wire. The avoiding of a (wire) crack is one of the the overall goals. The detection or prognosis of an upcoming (wire) crack is possible on different ways: On the one hand the measurement and analysis of the change of the electric resistance, on the other hand the measurement and determination of degradation of the stroke. Both measurements lead to time series data. The measurement of the electric resistance is an indirect indicator for an upcoming crack, because the resistance correlates with an decreasing (wire) cross section as a result of a beginning crack. But the resistance can be influenced also by changing of the material characteristics within a long-term use. Therefore the electrical resistance can be an crack indicator, but includes uncertainty. In this paper, the analytics of the time series data regarding the measurement of the stroke is in focus. The goal of the data analysis is the detection of a changing behaviour regarding the time series stroke data, which gives a hint to an upcoming collapse (crack) of the shape memory alloy element.

\section{Fundamentals}

For a better understanding of the case study as well as the applied statistical methods, the basics of the shape memory technology and the parameter-free methods are described within this chapter.

\subsection{Smart material alloy (actuators)}

This chapter is based on Heß and Bracke (2019) and Heß and Bracke (2018). Shape memory alloys (SMAs) have the ability to recover large deformations that can exceed the elastic deformability of the material significantly. The shape memory effect is used to restore a previously imprinted shape of components.

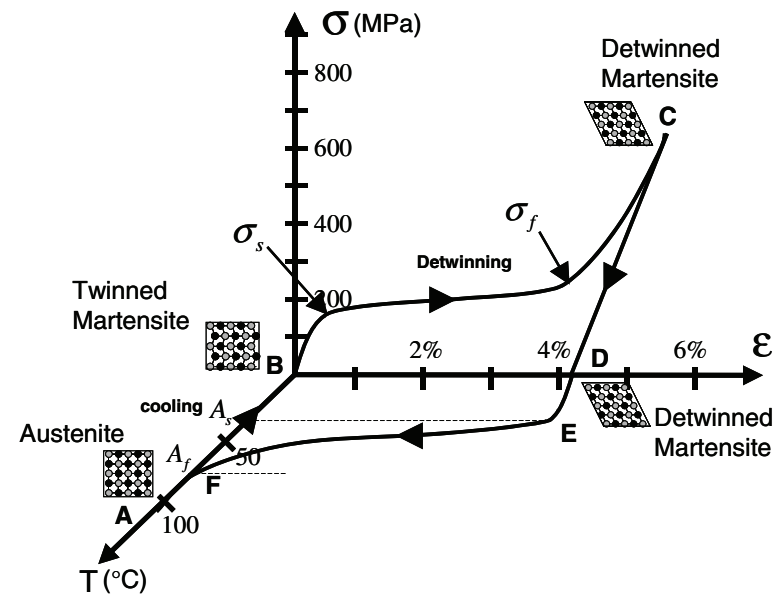

(a)

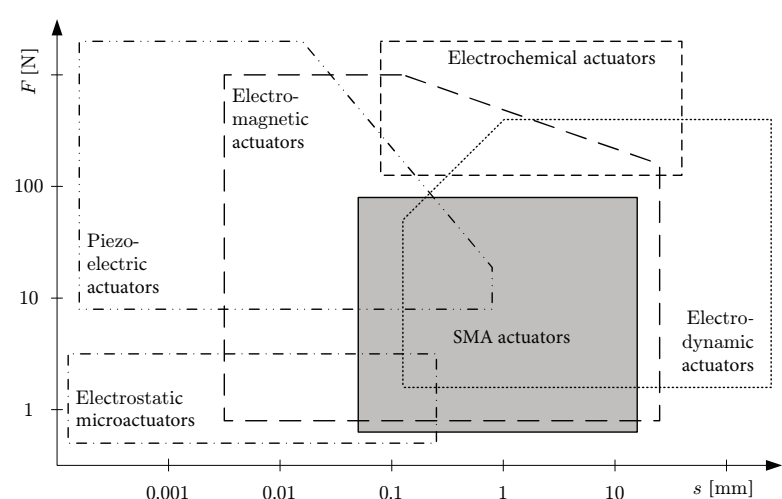

(b)

Source: (a) Lagoudas (2018); (b) cf. Schiedeck (2009)

Fig. 1 (a) SMA stress-strain-temperature diagram; (b) Possible fields of application for different technologies of actuators 
The material behavior relies on the martensitic phase transformation (Elahinia (2016), Heß and Bracke (2019), Heß and Bracke (2018), Hinz et al. (2018), Lagoudas (2008) and Rao et al. (2015)). Figure 1 (a) shows a stressstrain-temperature diagram of a typical SMA. Cooling the SMA below the martensite finish temperature $\left(M_{f}\right)$ causes the transformation from the high temperature austenite phase (A) into the low temperature martensite phase (B). The elastic deformation $(\epsilon)$ of the martensite phase results by applying stress $\left(\sigma_{\mathrm{s}}\right)$ at a constant temperature. Reaching the critical stress level $\left(\sigma_{\mathrm{f}}\right)$ results in the transformation to detwinned martensite $(\mathrm{C})$, while further loading induces a plastic deformation. A linear strain recovery (D) is achieved by unloading the SMA. Exceeding both, the austenite start (E) and finish (F) temperature, by heating the SMA (which can be achieved by thermal energy or electric heating), the material transforms back to austenite and the strain recovery is complete (A) (Elahinia (2016), Heß and Bracke (2019), Heß and Bracke (2018) and Lagoudas (2008)).

There are three possible effects of SMAs: The one-way effect, as described above, the two-way effect (pseudoplastic) and the superelasticity (pseudoelasticity). The effects can be used e.g. in actuators providing displacements and mechanical forces. Actual shape memory applications are thermal and electrical valves, release or actuating mechanisms and auto-focus systems of smartphone cameras (Heß and Bracke (2018) and Hinz et al. (2018) and Janocha (2010)). According to Elahinia (2016), Heß and Bracke (2019), Heß and Bracke (2018) and van Humbeeck (2001), the nickel titanium alloy with a ratio of 50:50 is the most common one, which is used for more than $90 \%$ of the actual commercial applications.

There are several advantages and disadvantages of smart material actuators compared to conventional actuators. The main advantages are simplification, miniaturization, noiseless operation, lightweight construction, high working capacity and an integrated sensory function. Despite those advantages, the major problem is the development time, due to the complex thermal behavior as well as fatigue behavior of shape memory actuators and applications. The resetting time that is referred to the cooling time from austenite finish temperature $\left(A_{f}\right)$ to martensite finish temperature $\left(M_{f}\right)$ is crucial for the suitability for the use in an application. The ambient temperature, the heat dissipation (e.g. housing of the actuator) and the usage of the actuator (e.g. temperature hysteresis caused by a high frequency) are the main influences regarding the cooling time (Heß and Bracke (2019)). Figure 1 (b) shows application possibilities of different actuator technologies. Each technology can be used for various required forces and displacements. There is a certain range for smart material actuators but the technology cannot be used to achieve high loadings, very large or small displacements (strokes) and high frequencies (Heß and Bracke (2019), Heß and Bracke (2018), Lagoudas (2008) and Schiedeck (2009)).

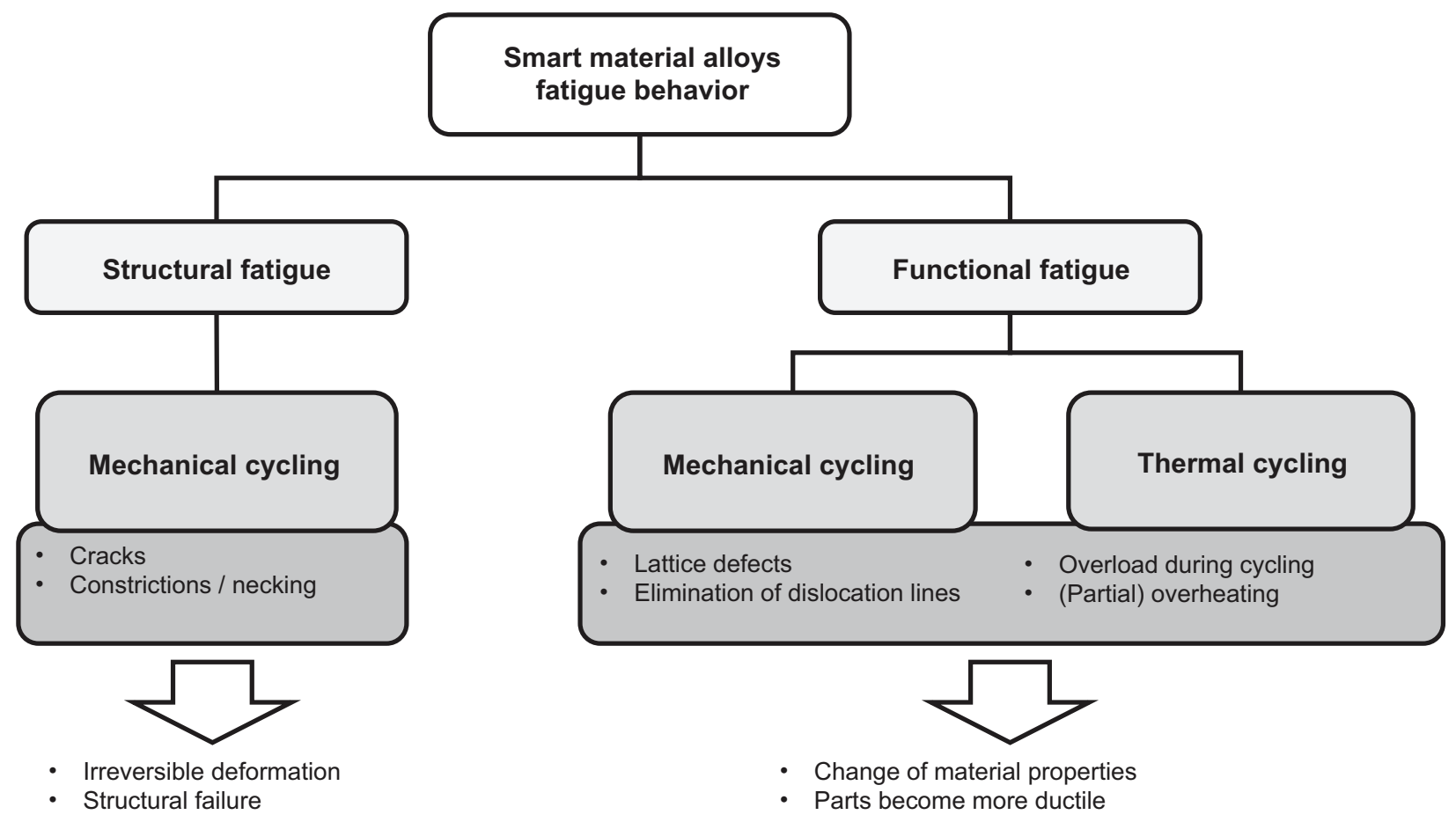

Source: c.f. Heß and Bracke (2018) and Czechowicz (2012)

Fig. 2 Fatigue behavior of smart material alloys 
The fatigue testing of smart material actuators is much more time consuming than testing of conventional actuators. Testing 150.000 cycles with a cycle time of about 20 seconds per cycle, can take up to 50 days. According to Heß and Bracke (2018), SMAs fatigue behavior can be divided into different types of fatigue, that are shown in Fig. 2. The functional fatigue behavior is significant for the reliability assessment. There are several influencing factors regarding the fatigue behavior: The material processing (e.g. the fabrication process, the surface finish quality or possible heat treatment), the loading conditions (e.g. the applied stress, strain and temperature) or possible transformation induced microstructural modifications (e.g. defects on grain boundaries caused by strain incompatibilities) (Heß and Bracke (2019), Heß and Bracke (2018), Hinz et al. (2018) and Lagoudas (2008)).

To determine and ensure the reliability of shape memory actuators, its fatigue behavior has to be analyzed in detail. Test rigs for this purpose cyclically activate multiple actuators with defined loadings and activation frequencies until failure. For the analysis of the test, all relevant parameters, e.g. time, voltage and current to activate the actuator, electrical resistance, displacement (stroke), ambient temperature, resetting force etc. are recorded. With a degrading stroke (displacement) of the actuator over the testing cycles it is possible to determine a dependency between actuator performance (maximum stroke) and fatigue. Ideally the dependency (degradation behaviour) can be used to estimate the lifetime of the actuator, which enables shorter test procedures (Heß and Bracke (2019)).

\subsection{Nonparametric statistics}

For small amounts of data (small sampling size) the use of parametric significance tests is not suitable. In addition, many industrially used parametric tests are generally based on the assumption, that the damage data / population follows the normal distribution. Based on the normally distributed population, all required parameters can be determined. But small sampling sizes only partially meet the assumption of normally distributed data. Because of these limitations of parametric significance tests regarding to an early statistical reliability analysis, other statistical methods have to be used to analyze prototype data. One approach is the use of nonparametric significance tests. Nonparametric tests do not require assumptions about the probability distribution of the variables to be examined. In comparison to similar application cases of parametric significance tests, they have also further application possibilities and benefits, in detail (Bracke and Haller (2011)):

- Applicable also for small sample sizes

- Application independent of distribution model

- Simple calculation

- Early comparison of individual tests to each other in relation to

- the centre of the failure cases and

- the dispersion of the failure cases

A good overview on relevant nonparametric significance tests is described in Büning (1994) and Siegel (2001). In the context of the application of nonparametric significance tests for analyzing (prototype) failure data in general only tests for independent samplings are suitable (Bracke and Haller (2011)). In the following some tests are exemplarily represented:

\section{One sampling case:}

Does the central tendency (e.g. median, dispersion etc.) concur (null hypothesis $H_{0}$ ) or differ (alternative hypothesis $H_{1}$ ) regarding to a hypothetical value (e.g. Sign-test):

$$
H_{0}: \Theta=\Theta_{0} ; \quad H_{1}: \Theta \neq \Theta_{0}
$$

The values of a time series are (not) subject to a trend (e.g. Cox and Stuart test):

$$
\begin{array}{lll}
H_{01}: P\left(y_{i}>0\right) \leq P\left(y_{i}<0\right) ; & & H_{11}: P\left(y_{i}>0\right)>P\left(y_{i}<0\right) \\
H_{02}: P\left(y_{i}>0\right) \geq P\left(y_{i}<0\right) ; & & H_{12}: P\left(y_{i}>0\right)<P\left(y_{i}<0\right) \\
H_{03}: P\left(y_{i}>0\right)=P\left(y_{i}<0\right) ; & & H_{13}: P\left(y_{i}>0\right) \neq P\left(y_{i}<0\right)
\end{array}
$$

\section{Two samplings case:}

Analysis of equality of two distributions $\mathrm{F}$ and $\mathrm{G}$ (null hypothesis $H_{0}$ ) (e.g. Wald-Wolfowitz runs test):

$$
H_{0}: F(z)=G(z) ; \quad H_{1}: F(z) \neq G(z)
$$


Two distributions $\mathrm{F}$ and $\mathrm{G}$ have the same shape, but differ in a location parameter $\Theta$ (alternative hypothesis $H_{1}$ ) (e.g. Mann-Whitney u-test and Wilcoxon rank sum test):

$$
H_{0}: G(z)=F(z) ; \quad H_{1}: G(z) \neq F(z-\Theta)
$$

Two distributions $\mathrm{F}$ and $\mathrm{G}$ differ regarding to variability (alternative hypothesis $H_{1}$ ) (e.g. Levene's test and Siegel-Tukey test):

$$
H_{0}: G(z)=F(z) ; \quad H_{1}: G(z) \neq F(\Theta z)
$$

\section{C samplings case:}

$\mathrm{C}$ distributions have the same shape, but differ in a location parameter $\Theta$ (alternative hypothesis $H_{1}$ ) (e.g. Kruskal-Wallis test):

$$
H_{0}: F_{1}(z)=F_{2}(z)=\ldots=F_{c}(z)=F(z) ; \quad H_{1}: F_{i}(z) \neq F\left(z-\Theta_{i}\right)
$$

C distributions differ in the variability (alternative hypothesis $H_{1}$ ) (e.g. Meyer-Bahlburg test (generalised Siegel-Tukey test)):

$$
H_{0}: F_{1}(z)=F_{2}(z)=\ldots=F_{c}(z)=F(z) ; \quad H_{1}: F_{i}(z) \neq F\left(\Theta_{i} z\right)
$$

C samples (distribution function F, median) are (not) subject to a trend (e.g. Jonckheere-Terpstra test)):

$$
\begin{array}{ll}
H_{0}: F_{1}=F_{2}=\ldots=F_{k} ; & \mu_{1}=\mu_{2}=\ldots \mu_{k} \\
H_{1}: F_{1} \leq F_{2} \leq \ldots \leq F_{k} ; & \mu_{1} \leq \mu_{2} \leq \ldots \mu_{k} \\
H_{2}: F_{1} \geq F_{2} \geq \ldots \geq F_{k} ; & \mu_{1} \geq \mu_{2} \geq \ldots \mu_{k}
\end{array}
$$

This paper is focused on the Levene's test, the Mann-Whitney u-test, the Cox and stuart test and the JonckheereTerpstra test.

\section{Case study}

This case study deals with three different endurance tests, which are all performed on the same test rig. Each test consists of six actuators. The test rig, the actuators and the measurement data represent the basis for the analysis presented in chapter 5 and will be explained as follows. The description is based on Heß and Bracke (2019).

\subsection{Test rig}

The test rig of the case study, shown in Fig. 3 (a) is designed for testing up to six actuators, which use a spring as the resetting element. The actuators consist of a fixed bearing and a moveable bearing which are centered by a pin. The spring pushes the moveable bearing against a stopper. Four pairs of SMA wires are wrapped through grooves of the bearings and are fixed with splice connections, which are standarized by VDI (2017). Furthermore, the different wire pairs are connected at the bottom by circuit boards and a power source. The test rig is equipped with a displacement sensor for each actuator and a temperature sensor. The temperature sensor measures the ambient temperature at the middle position on the top of the test rig.

During the endurance test, the actuator is cyclically stressed with defined parameters (e.g. resetting force, voltage to activate, ambient temperature etc.). The actuator is activated by a current pulse (rectangular shape) which heats up the SMA wires causing the shape change to its previously imprinted shape. Working against the force of the spring, the wires pull down the moveable bearing. During the cooling time of the wires, the spring pushes the bearing back against the stopper to its initial position. Figure 3 (b) shows an exemplary recorded measurement time series of stroke and voltage of one actuator. In this example, nearly seven activations of the actuator are performed within 120 seconds. Activating the actuator with a current pulse initiates the stroke immediately. The stroke peaks at about $3.2 \mathrm{~mm}$, the current pulse at about 6.8 V. Compared to the cooling phase, the activation is much faster. The test sequence is controlled by a process control unit in such way that a cycle is started without pausing after the previous one finished. 


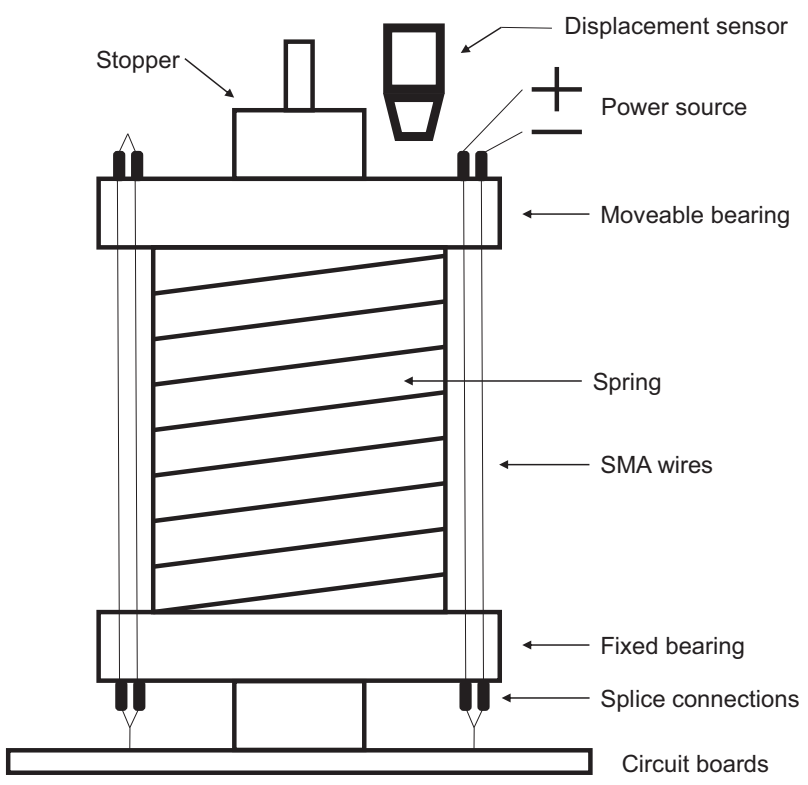

(a)

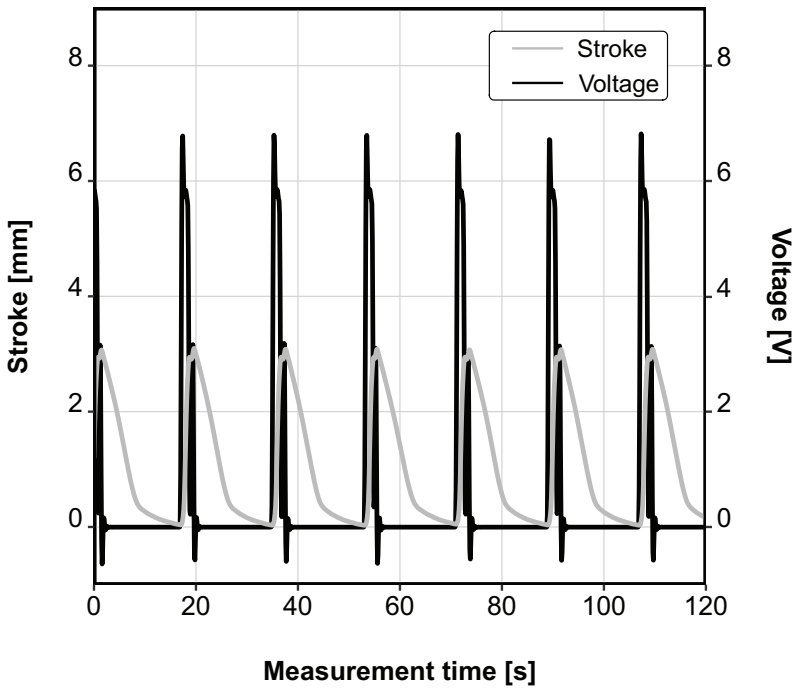

(b)

Source: $(a, b)$ Heß and Bracke (2019)

Fig. 3 (a) Sketch of principle of a test rig for shape memory alloy actuators which use a spring as resetting element; (b) Measurement series of a test rig showing the recorded variables stroke $[\mathrm{mm}]$ and voltage for activation [V] over time [s]

\subsection{Measurement data}

With the test rig measurement time, displacement (stroke), voltage and current (for activation), number of cycles and ambient temperature of six actuators of the principle as shown in Fig. 3 (a) are recorded. The electrical resistance can be calculated using the parameters voltage and current. The test rig records data without pausing, therefore each activation is documented in detail. The extraction of each peak value of the fatigue test provides an initial overview of the stroke behavior over the complete duration of the test.

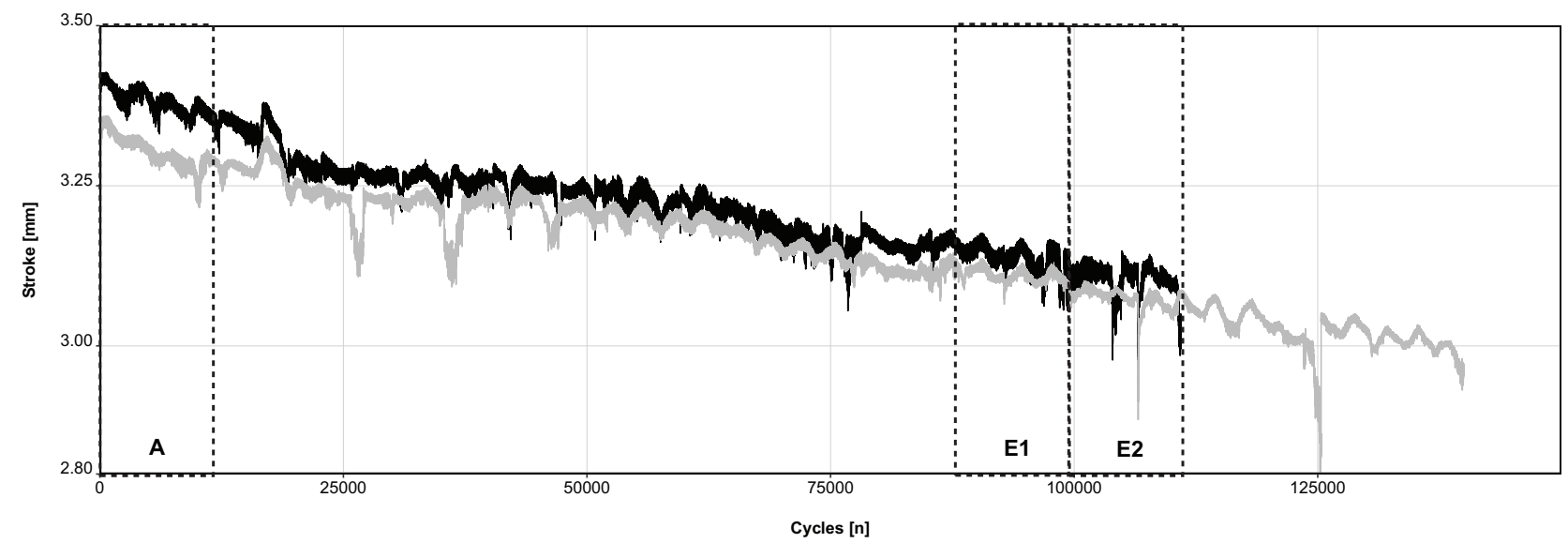

Fig. 4 Full endurance test of 2 actuators of test 1: Stroke peaks [mm] over time

The diagram, shown in Fig. 4, illustrates the stroke behavior of two actuators of test 1. A clear decrease of the stroke from about $3.3 \mathrm{~mm}$ respectively $3.4 \mathrm{~mm}$ to $3.1 \mathrm{~mm}$ can be observed over the test period. The remaining actuators, comming from the same test rig, show a similar course of the measured values. Test 2 and 3 also show a similar degradation process. It is noticeable that some actuators of all three tests additionally show the same behavior in detail (similar waveforms). The (nonparametric) analysis in this paper is focused on the areas A, E1 and E2 (shown in Fig. 4), as this allows a comprehensive comparison of the start of the test and the area before and during the actuator failures. 
For a deeper understanding of the experiments, the general similarities and differences are discussed respectively. Within all three experiments, a total of six identical actuators with an activation voltage of $6.6 \mathrm{~V}$ have been tested with the same setup. Parallel to those similarities, the three tests differ in detail, as shown in table 2.

Table 2 Overview of the different test conditions and test sequences regarding 3 tests each containing 6 actuators

\begin{tabular}{r|c|c|c|c}
\hline Parameter & Test 1 & Test 2 & Test 3 & Unit \\
\hline Current & 2.3 & 3.1 & 3.1 & $\mathrm{~A}$ \\
\hline Initial stroke & 3.2 & 3.2 & 1.8 & $\mathrm{~mm}$ \\
\hline Cycles total & 139.000 & 200.000 & 158.000 & $\mathrm{n}$ \\
\hline First break & 110.000 & none & 25.000 & $\mathrm{n}$ \\
\hline Other breaks & 139.000 & none & none & $\mathrm{n}$ \\
\hline Mean temperature & 32.2 & 30.3 & 26.4 & ${ }^{\circ} \mathrm{C}$ \\
\hline Minimum temperature & 26.2 & 26.9 & 24.2 & ${ }^{\circ} \mathrm{C}$ \\
\hline Maximum temperature & 36.9 & 33.4 & 31.3 & ${ }^{\circ} \mathrm{C}$ \\
\hline
\end{tabular}

Within test 1, an initial maximum stroke value of $3.2 \mathrm{~mm}$ was observed (current for activation = 2.3 A). All SMA wires break after a certain amount of cycles. Actuator 2 breaks after about 110.000 cycles, while the others break almost at the same time after approximately 139.000 cycles. The ambient temperature during the entire test has a mean value of $32.2^{\circ} \mathrm{C}$ (maximum $=36.9{ }^{\circ} \mathrm{C}$, minimum $=26.2^{\circ} \mathrm{C}$ ). The second test has an increased current for activation, but the same initial stroke values. The temperature is slightly lower compared to the first test. The test was aborted after about 200.000 cycles without a single actuator fault. It is unclear how long the experiment could have been continued. Test 3 was also carried out with a higher current, but has a lower initial stroke value. The ambient temperature was also lower during the whole endurance test. Relatively early, after roughly 25.000 cycles, the first actuator of this experiment failed. The other 5 actuators were tested until termination at about 158.000 cycles. As with the second experiment, it is also unclear how long the experiment could have been carried out.

\section{Analysis of test rig data and discussion of the results}

This chapter provides an overview of models developed and methods applied so far, as well as new evaluations related to a project on shape memory actuators. Due to the scope, all analyses, including the new ones, are usually only presented as examples. For more detailed information on the methods and analyses already published, see Heß and Bracke (2019) and Heß and Bracke (2018).

The measured data of the endurance tests is prepared and analyzed using different procedures, techniques and statistical methods. According to Heß and Bracke (2019) data cleansing is the first necessary step to achieve a data set of the case study that allows a precise analysis. The Hampel filter defined in Pearson (1999) is used to identify median absolute deviation (MAD) outlier in time series. The parameters of the filter are selected in such a way that the data of the case study is just smoothed filtering very large outliers. The measurement data is influenced just as much as needed. The data is then prepared for the analysis. The maximum of all measured process parameters (stroke, voltage, current, resistance and temperature) for every actuator is determined for each cycle of all three tests. Due to the fact that in the first test actuator 2 fails much earlier than the others, the Levene's test and the u-Test by Wilcoxon and Mann-Whitney are performed to assess the equality of variances and mean values between two actuators of the areas $A, E 1$ and E2 (shown in Fig. 4). In Fig. 5, the corresponding box-plots of actuator 1 (a) and actuator 2 (b) can be observed. Depending on the test, the prepared data set contains 139.000 to 200.000 cycles, each of those corresponds to a value for the recorded parameters. For all nonparametric statistical analyses, the amount of data is reduced by using only every 10 th value for the analysis. The size of the areas $A, E 1$ and $E 2$ is selected in such way, that the samples contain 1.000 values. The u-test shows that the null hypotheses are rejected. However, a larger difference of the mean values of area $A$ and the areas E1 / $E 2$ can be determined for actuator 2 (b), which suggests a higher slope of the regression line. The variance of the ranges of area $E 1$ and $E 2$ of actuator 2 is also significantly lower than that of actuator 1. The comparison of actuator 2 to the other actuators of this test shows similar results. It should be noted that only the data up to cycle 110.000 of both actuators was used to compare the areas A, E1 and E2 of both measurement series. 


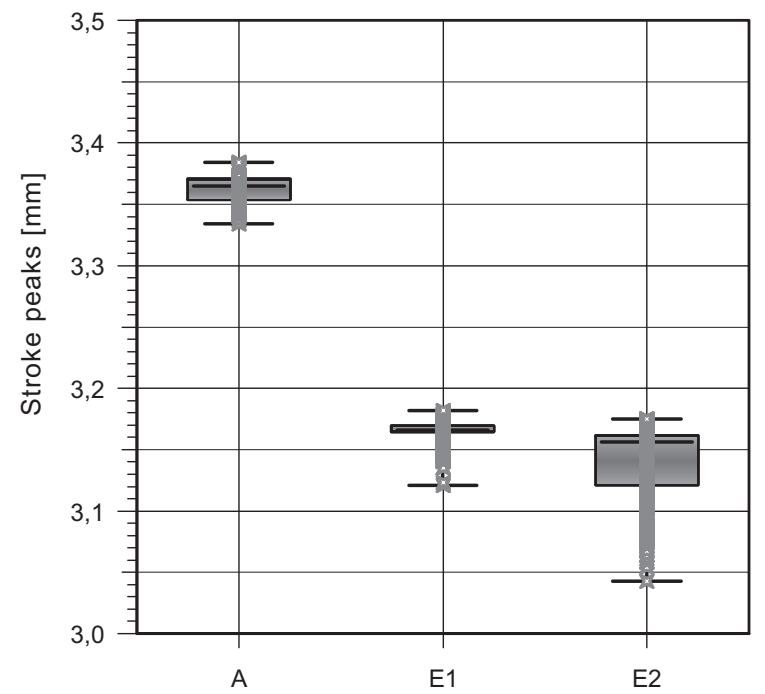

(a)

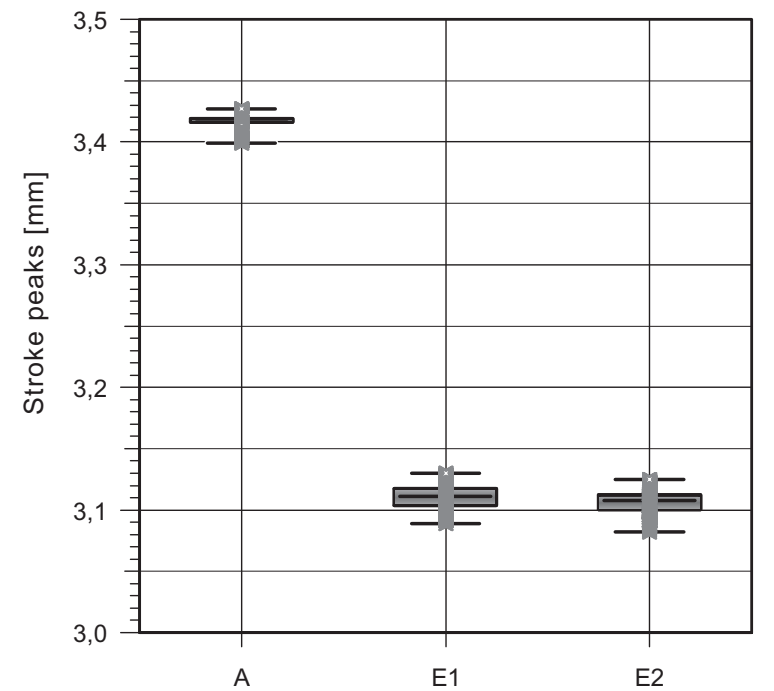

(b)

Fig. 5 Box-plots of the stroke values of area $A, E 1$ and $E 2$ of actuator 1 (a) and actuator 2 (b) of test 1

Since an actuator also broke in test 3, the same procedure can be used for this test. For this purpose again, only the data up to the breakage of actuator 1 (about 25.000 cycles) of both actuators was used here again. The result is displayed in Fig. 6 comparing the broken actuator 1 (a) to actuator 4 (b). The comparisons of actuator 1 to other actuators of the same test are completely different, which can be explained by the fact that the number of values is not large enough (only 25.000 cycles).

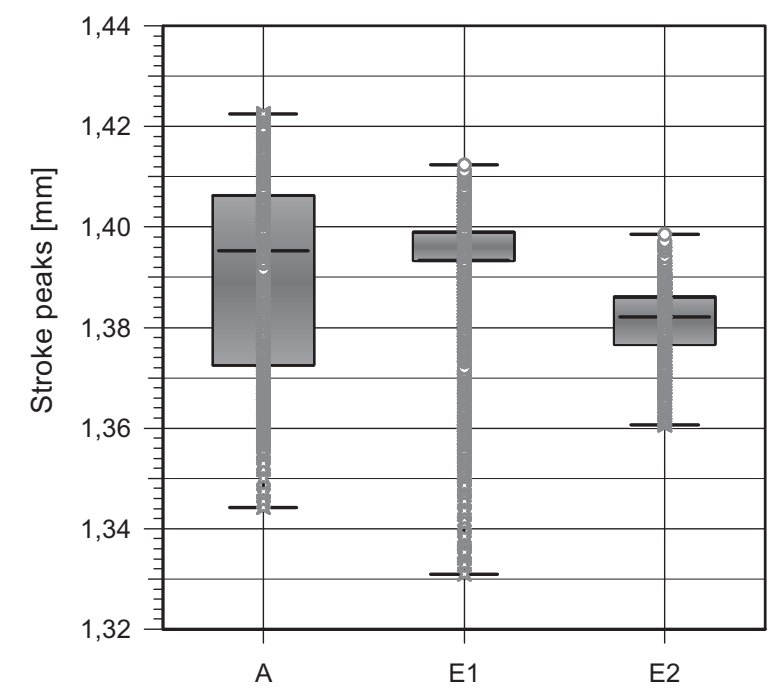

(a)

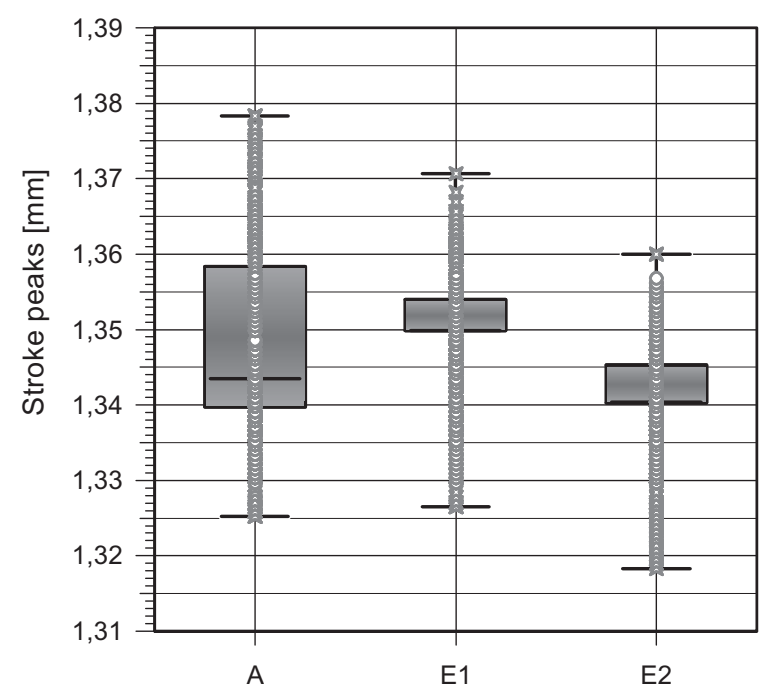

(b)

Fig. 6 Box-plots of the stroke values of area $A, E 1$ and $E 2$ of actuator 1 (a) and actuator 4 (b) of test 3

Another crucial difference between the different tests is the average ambient temperature. A possible influence of the temperature on the maximum stroke or the stroke and the degradation behavior of an actuator is also analyzed by the Levene's test and the u-Test by Wilcoxon and Mann-Whitney. First, the gradients of the regression line of each actuator of all 3 tests are determined. For this purpose, the value range does not correspond to the ranges $A, E 1$ and E2, but to the entire and unreduced measurement series up to the individual failure or up to the termination of the test. The nonparametric test uses 3 samples, each containing 6 values. By using the gradient, a comparison of all 3 tests is possible, even if test 3 has a different initial stroke value. 
The results are shown in Fig. 7 (a). For a better understanding of the relationship between the stroke characteristics and the ambient temperature, Fig. 7 (b) illustrates the profile of the maximum temperature as an example for Test 1.

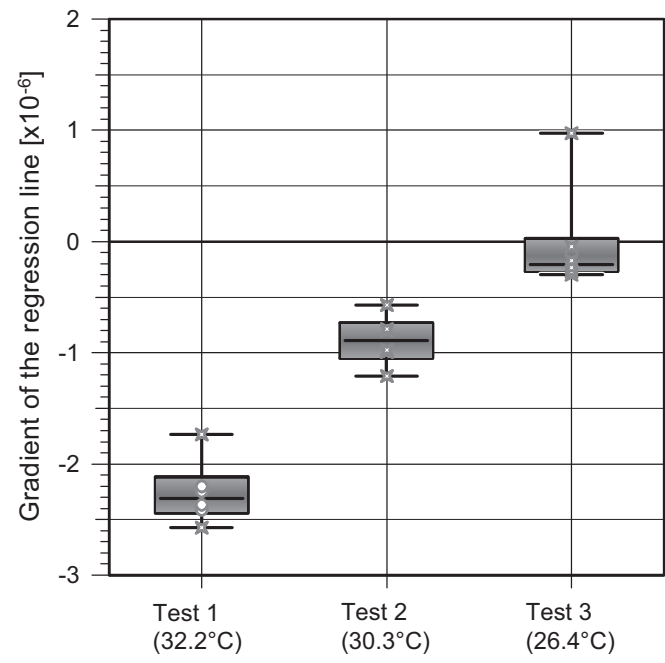

(a)

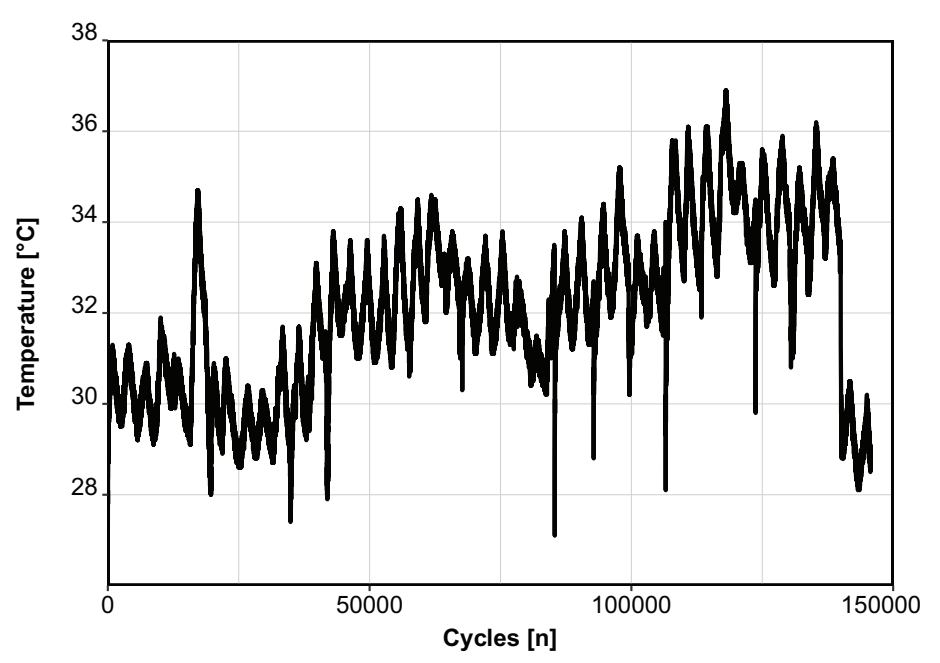

(b)

Fig. 7 (a) Box-plots of the gradients of the regression lines of all actuators of test 1 to 3;

(b) Temperature profile of test 1

The nonparametric tests show that the null hypothesis that the mean values are equal is rejected, but the null hypothesis that the variances are equal is not rejected. It can be clearly seen that the gradient of the regression line decreases if the average as well as the maximum ambient temperature were lower during the test (see table 2). The JonckheereTerpstra test returns the following results: The Hypothesis $H_{0}$ that the three samples are not following a trend is rejected $\left(\mathrm{p}\right.$-value $\left.=1.166 e^{-07}\right)$. Furthermore the alternative Hypothesis that the samples follow an increasing trend $H_{1}$ is accepted $\left(\mathrm{p}\right.$-value $=5.83 e^{-08}$ ), while the alternative Hypothesis that the samples follow a decreasing trend $H_{2}$ is rejected ( $\mathrm{p}$-value $=1$ ). However, there is no temperature influence on high fluctuations in the degradation process, since the variances are roughly the same in all temperature ranges.

A significant influence of the ambient temperature on the stroke behavior was also analyzed regarding another case study ( $\mathrm{He} ß$ and Bracke (2018)). Figure 8 shows the comparison of normalized values (all values between 0 and 1) of stroke and ambient temperature.

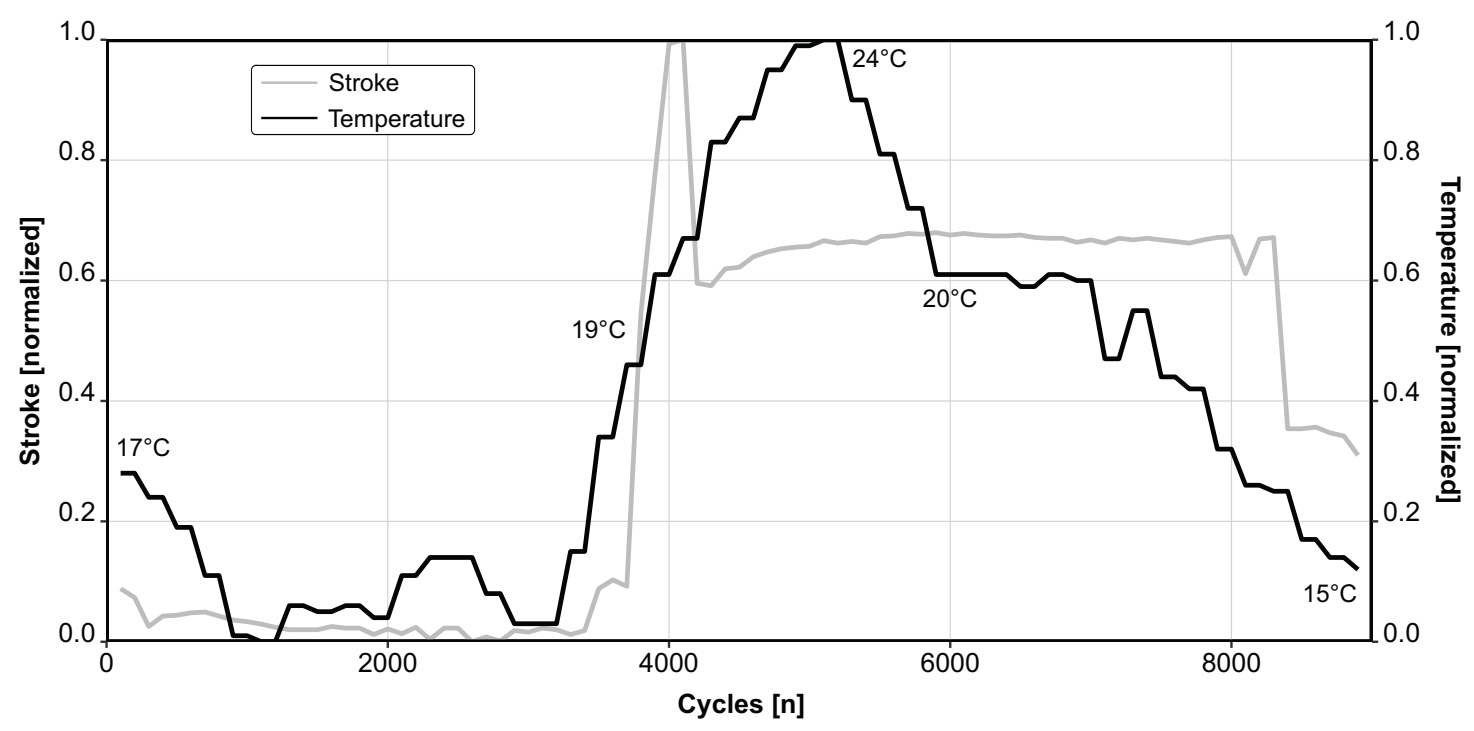

Source: c.f. Heß and Bracke (2018)

Fig. 8 Case study "FGL-ZZ": Stroke and temperature (normalized) 
The temperature values are weather data of the nearest weather station (approx. $5 \mathrm{~km}$ distance) as the test bench does not have a temperature sensor. In order to further analyze the temperature influence in detail as well as dependencies of the measured variables, a Spearman correlation analysis is applied, which according to Sachs (1984) describes the linear relationship between 2 variables. A strong correlation can be observed in case of temperature and stroke with a coefficient of about 0.8 . The coefficient of the tests of the case study of this contribution is between 0.6 and 0.8 for all actuators.

The next goal of the evaluations is the prediction of the failure behavior. The following regression analysis is shown in a short form (for further information regarding this regression analysis as well as the model for the prognosis of the lifetime refer to Heß and Bracke (2019)).

The first step is a goodness of fit test (e.g. the Kolmogoroff-Smirnoff test) to estimate a suitable model for the regression analysis. According to Birolini (1999) and Sachs (1984), the Kolmogoroff-Smirnoff test is used to quantify the goodness of fit of a data set (e.g. the stroke values) to a given distribution (e.g. a normal distribution). The stroke values can be assigned to a mixed distribution. Considering the mixed distribution, a generalized linear model for regression is applied. For this purpose, the Ordinary Least Squares (OLS) method, as described in Gourieroux (1997) and Hutcheson (2011), is applied to estimate the parameters of the linear regression model by minimizing the sum of squared distance between the observed values (maximum stroke) and the corresponding fitted values.

To determine how many test cycles are necessary to predict the failure time with an accuracy above $90 \%$, the measurement series is subdivided into increasing parts (sections, ranges), in which only the testing range until failure is considered. The first part consists of the first 1.000 cycles, the second section consists of the first 2.000 cycles etc. Finally the 130.000 cycles of the fatigue test result in 130 parts, each increased by 1.000 cycles. A regression analysis is performed for each part. The regression lines are determined using the straight line equation. Since the regression line of part one only covers the first 1.000 cycles, it must be extended to the cycle of failure by a suitable prediction model, as well as the other parts. The developement of the stroke over time within the fatigue test with the according regression lines of two different parts and of the full test are exemplary shown in Fig. 9 (a).

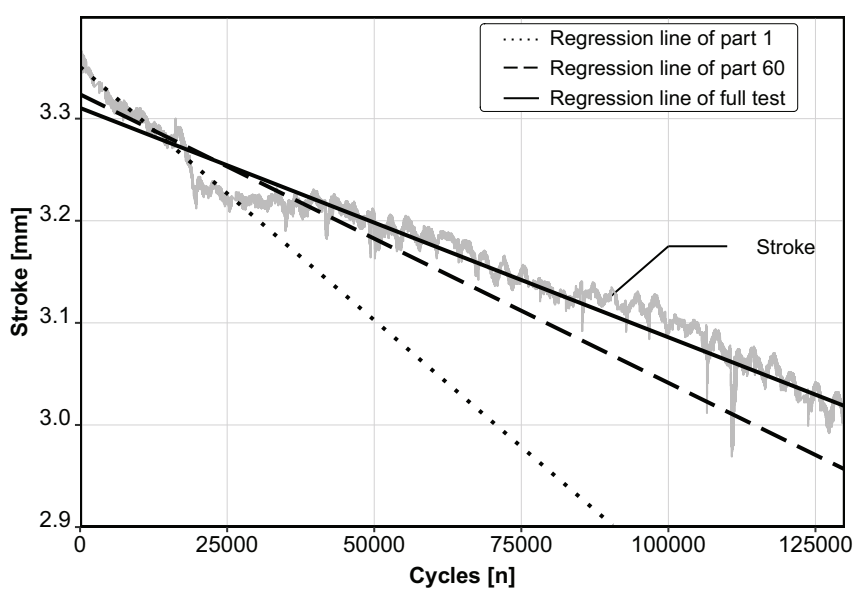

(a)

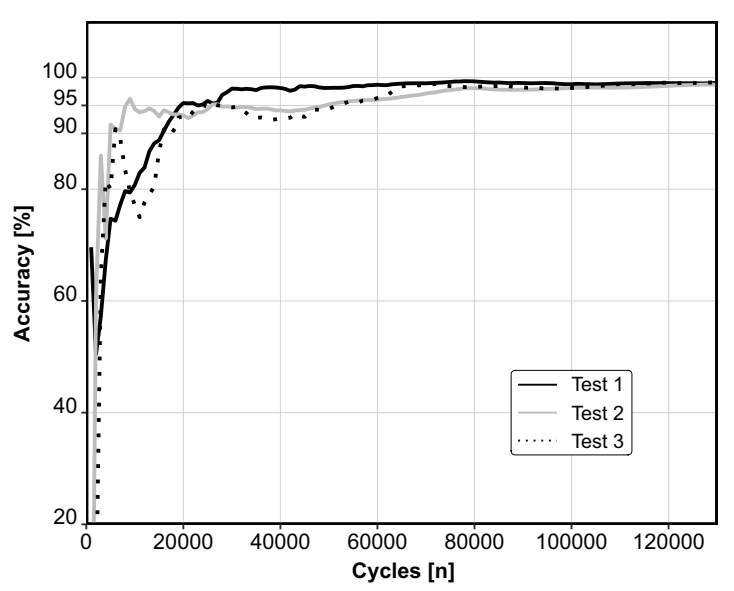

(b)

Source: (a) Heß and Bracke (2019)

Fig. 9 (a) Fatigue test 1: Real data of 1 actuator and the fit regression models; (b) Average accuracy [\%] for all 3 tests depending on the section size

The predicted end points of the regression lines $Y_{\text {predicted }}$ are compared with the real value $Y_{\text {failure }}$, which is measured at the time of failure (reference value). The percentage of accuracy $Z_{Z}$ is calculated using Eq. (1), the graphical representation is shown in Fig. 9 (b). For tests 2 and 3, $Y_{\text {failure }}$ was defined as the end of the test for the non-defective actuators. The consistency converges differently for the test benches within the first 50.000 test cycles. Almost no difference between the test benches can be determined above 60.000 cycles. As a result, it can be stated regarding the study that the value at the time of failure $Y_{\text {failure }}$ can already be predicted after 60.000 cycles (less than $50 \%$ of total cycles) with an accuracy of more than $90 \%$.

$$
Z_{Z}=1-\frac{\left|Y_{\text {predicted }}-Y_{\text {failure }}\right|}{Y_{\text {failure }}} \times 100
$$


The last method to analyze the test rig data, that is exemplary presented in this article, is the usage of multivariate plots. It is possible to show 3 or more variables with these 3D-plots, allowing potential dependencies to be analyzed (in detail). Figure 10 shows the multivariate 3D-plot of actuator 1 of test 1 from different viewpoints, including the parameters stroke $(\mathrm{Y})$, current for activation $(\mathrm{X})$ and ambient temperature $(\mathrm{Z})$.

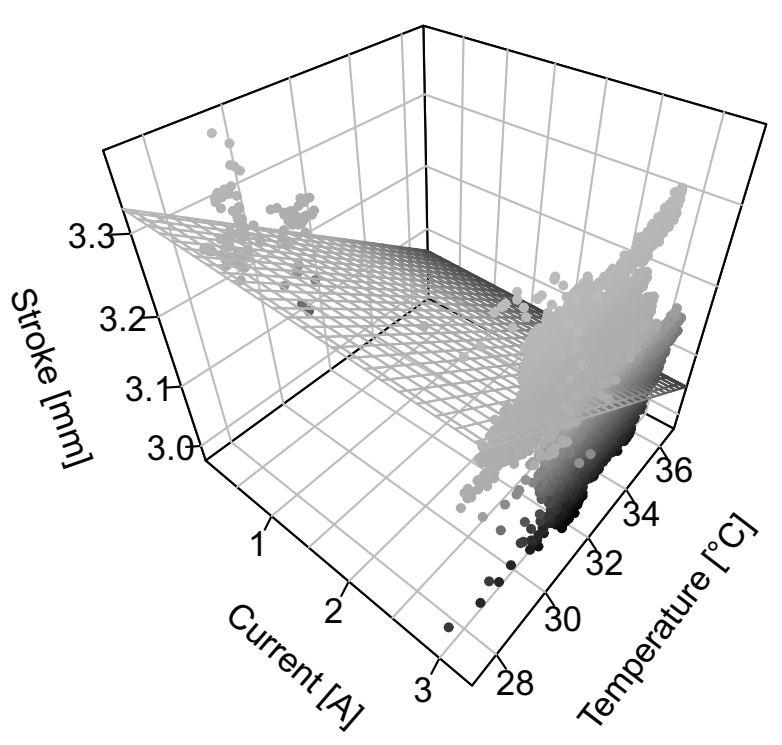

(a)

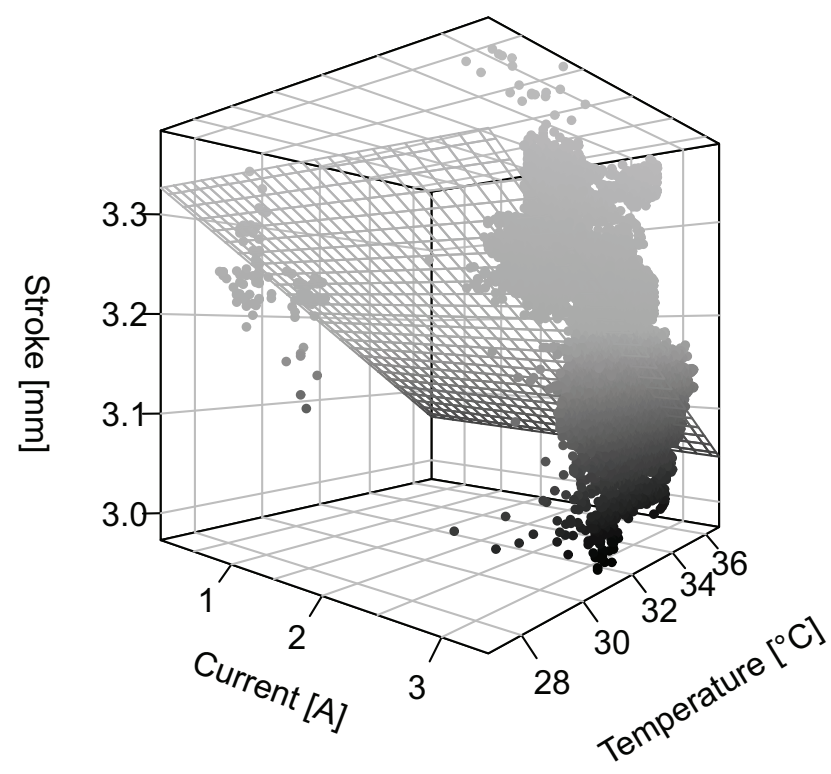

(b)

Fig. 10 3D-plot (different perspectives) of actuator 1 of test 1: Stroke [mm], current for activation $[\mathrm{A}]$ and ambient temperature $\left[{ }^{\circ} \mathrm{C}\right]$

The plot clearly shows the influence of the ambient temperature on the actuators or rather the wires. At higher temperatures, the maximum stroke values of the actuator are lower. Within the temperature range from $28{ }^{\circ} \mathrm{C}$ to $36{ }^{\circ} \mathrm{C}$, the stroke value decreases by almost $0.3 \mathrm{~mm}$ (from over $3.3 \mathrm{~mm}$ to $3.05 \mathrm{~mm}$ ). Furthermore, it can be observed that there are a few activations where only a current of about $1 \mathrm{~A}$ was applied for the activation, which could possibly attributed to measurement errors. The majority of all cycles were activated with 3 A. As already described with previous methods, a temperature influence on the stroke behavior of shape memory actuators can be determined.

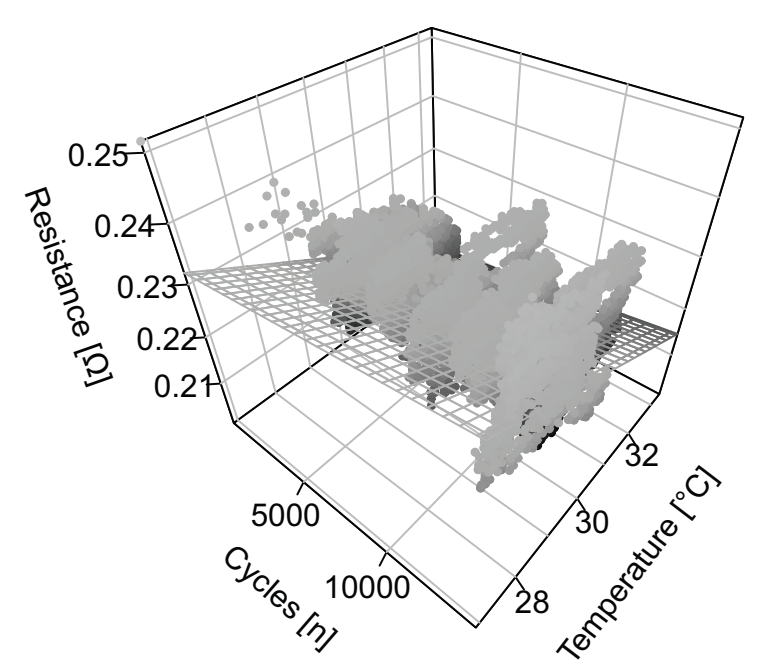

(a)

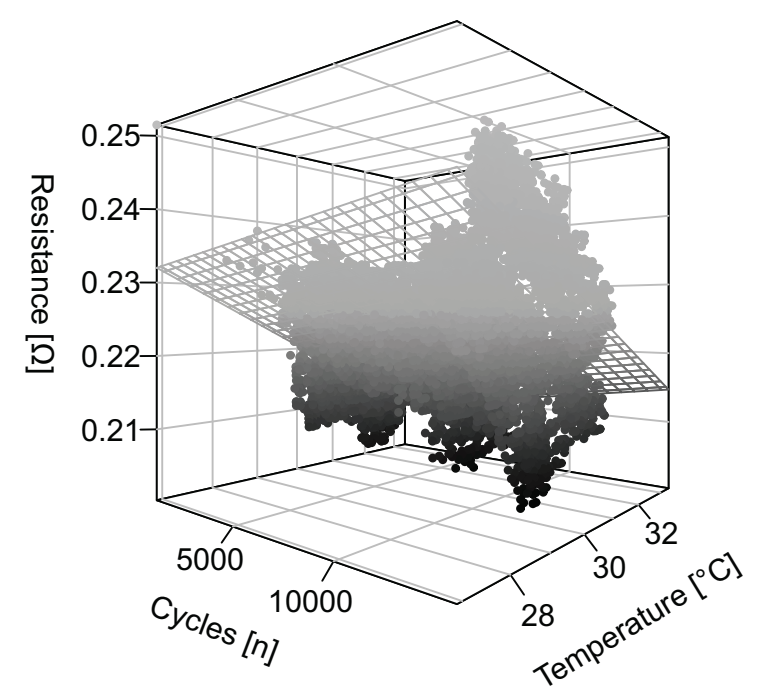

(b)

Fig. $113 \mathrm{D}$-plot (different perspectives) of actuator 1 of test 2: Wire resistance $[\Omega]$, cycles $[\mathrm{n}]$ and ambient temperature $\left[{ }^{\circ} \mathrm{C}\right]$ 
Since temperature changes have an influence on the resistance of shape memory alloys, it is obvious to analyze this influence further. Figure 11 shows a 3D-plot of wire-resistance (Y) and ambient temperature (Z) over all test cycles (X) of actuator 2 of test 2 . The plot illustrates that the wire resistance is slightly increasing over the test cycles. However, a much greater influence on the resistance can be observed at ambient temperature. Within the temperature range of $4{ }^{\circ} \mathrm{C}$ $\left(28^{\circ} \mathrm{C}\right.$ to $32{ }^{\circ} \mathrm{C}$ ), there is a tendency for the resistance to increase by $0.04 \Omega(0.21 \Omega$ to $0.25 \Omega$ ).

\section{Summary and outlook}

Shape memory actuators are particularly well suited for the use in IoT-based systems because they can also be used as sensors at the same time. The fact that these actuators can be monitored simultaneously results in advantages such as reduced installation space. But, for IoT-based systems, it is essential that all interacting components function reliably at all times. This article presents several methods for the analysis of test rig data of smart material actuators with the purpose to determine possible dependencies between the recorded parameters as well as an approach for a regression model to predict the lifetime of shape memory actuators at an early stage of a fatigue test. For an understanding of the complex technology, its fatigue behavior and usage in actuators as well as the necessary fundamentals of shape memory alloys are described briefly. Furthermore the actuator type, the test rig of the endurance tests, the testing process and the measurement data of the tests of a case study are described in detail. Finally, three tests, each including six actuators, are analyzed using several nonparametric tests and (statistical) methods. The final result of the analysis shows, that differences between failed and non-failed actuators can be detected and that the ambient temperature has a large influence on the resistance and the stroke behavior of the actuators. The prediction model is applied to the measurement data of the three tests. The determination of the accuracy between the values at time of failure and the predicted values shows that a prognosis with an accuracy over $90 \%$ is possible using less than the first $50 \%$ of the measured cycles.

Nevertheless, the actual reasons for the failure have to be analyzed further, which means that the time of failure itself cannot be predicted yet. In the future work the methodical procedure will be extended by further methods and techniques. New test rigs will be equipped with additional sensors (e.g. a force sensor), to analyze further influencing variables and interactions.

\section{Acknowledgements}

The authors thank Forschungsgemeinschaft Werkzeuge und Werkstoffe e.V. - FGW (Remscheid, Germany) for providing test rig data. Furthermore the authors thank the project sponsor Federal Ministry for Economic Affairs and Energy - BMWI (Germany) for funding and supporting this project.

\section{References}

Bertsche, B., Reliability of mechatronic systems: Basics and evaluation in early development phases, (2009), Berlin: Springer (in german).

Birolini, A., Reliability engineering: Theory and practice (3. ed.), (1999), Berlin: Springer.

Bracke, S. and S. Haller, Early identification of manufacturing process influences on product failure behaviour based on small field data volume. 4th International Conference on Changeable, Agile, Reconfigurable and Virtual Production (CARV 2011), Montreal, 2nd - 5th October, Canada 2011. In: Enabling Manufacturing Competitiveness and Economic Susbinability, (Editor: H. El Maraghy), 2012, CIRP, Springer.

Büning, H. and G. Trenkler, Nonparametric statistical methods, (1994), Berlin: De Gruyter (in german).

Czechowicz, A., Adaptive and adaptronic optimization of shape memory actuator systems for automotive applications, (2012), dissertation University of Bochum (in german).

Elahinia, M. H. (Ed.), Shape Memory Alloy Actuators: Design, Fabrication, and Experimental Evaluation (1. ed.), (2016), Wiley.

Gourieroux, C. and A. Monfort, Time series and dynamic models: Themes in modern econometrics, (1997), Cambridge: Cambridge Univ. Press.

$\mathrm{He}$, P. and S. Bracke, Reliability and degradation analysis of smart material actuators, (2019), Proceedings of the 29th European Safety and Reliability Conference, 22nd - 26th September 2019, Hannover, Germany (paper accepted).

$\mathrm{He}$, P. and S. Bracke, Reliability of shape memory actuators: Development of test bench technology and test program, (2018), GQW-conference 2018 (in german). 
Hinz, M., A. Czechowicz, D. Brüggemann, P. Dültgen and S. Bracke, Concept development for a test rig and analysis of the experiments for standardized testing of shape memory alloys, (2018), PSAM 2018 - Probabilistic Safety Assessment and Management.

Hutcheson, G.D. and L. Moutinho, The Sage dictionarry of quantitative management research, (2011), Los Angeles: Calif and London: SAGE.

Janocha, H., Unconventional actuators: An introduction, (2010), Munich: Oldenbourg (in german).

Kouicem D. E., A. Bouabdallah, H. Lakhlef: Distributed Fine-Grained Secure Control of Smart Actuators in Internet of Things, (2017), 2017 IEEE International Symposium on Parallel and Distributed Processing with Applications and 2017 IEEE International Conference on Ubiquitous.

Lagoudas, D. C., Shape memory alloys: Modeling and engineering applications, (2008), Boston, MA: Springer US.

Meeker, W. Q. and L. A. Escobar, Statistical methods for reliability data, (1998), Wiley series in probability and statistics: Applied prbability and statistics section, New York: Wiley.

Nelson, W., Accelerated testing: Statistical models, test plans and data analysis, (2004), Wiley series in probability and mathematical statistics: Applied probability and statistics, Hoboken, NJ: Wiley-Interscience.

Pearson, R. K., Data cleaning for dynamic modeling and control, (1999), 1999 European Control Conference (ECC).

Rao, A., A. R. Srinivasa and J. N. Reddy, Design of shape memory alloy (SMA) actuators, (2015), Springer Briefs in Applied Sciences and Technology, Cham: Springer International Publishing.

Sachs, L., Applied statistics: A handbook of techniques (2. ed.), (1984), Springer series in statistics, New York: Springer.

Schiedeck, F., Development of a model for shape memory actuators in controlled dynamic operation, (2009), dissertation, University of Hannover (in german).

Sendler, U., The Internet of Things: Industrie 4.0 Unleashed, (2019), Berlin Heidelberg (Germany): Springer Vieweg.

Siegel, S., Nonparametric statistical methods, (2001), Frankfurt: Klotz (in german).

van Humbeeck, J., Shape memory alloys: A material and a technology, (2001), Advanced Engineering materials 3.

Verband der Automobilindustrie e.V. (VDA), Quality management in the automotive industry (Volume 3, Part 2): Reliability assurance for automobile manufacturers and suppliers (3. ed.), (2000), Frankfurt: VDA.

Verein Deutscher Ingenieure (VDI), VDI 2248: Product development with shape memory alloys, (2017), (in german). 